\title{
Quantum paradigms in psychopathology: multiscale investigations from biomolecular qubits to the brain, and its pathological states
}

\author{
Massimo Cocchi ${ }^{1,{ }^{\dagger}}$, Marco Deriu $^{2,{ }^{\dagger}}$, Jack A. Tuszynski ${ }^{2,3, *,+}$ \\ ${ }^{1}$ Department of Veterinary Medical Sciences, Università di Bologna, 40126 Bologna, Italy \\ ${ }^{2}$ DIMEAS, Politecnico di Torino, 0137 Turin, Italy \\ ${ }^{3}$ Department of Physics, University of Alberta, Edmonton, AB T6G, Canada \\ *Correspondence: jackt@ualberta.ca (Jack A. Tuszynski) \\ ${ }^{\dagger}$ These authors contributed equally.
}

DOI:10.31083/j.jin2004112

This is an open access article under the CC BY 4.0 license (https://creativecommons.org/licenses/by/4.0/).

Submitted: 2 July 2021 Revised: 11 July 2021 Accepted: 12 July 2021 Published: 30 December 2021

The issue of the molecular nature of brain activities has been of great interest to not only neurophysiologists but also information scientists and psychopathologists. While much is known about the architecture of brain cells and their individual activities in terms of the action potential, ion channels and ionic currents, much less is known about such issues as where are memories stored, which molecular mechanisms are involved in information processing and cognitive functions. Speculations about molecular mechanisms of cognition range from mundane (mechanistic) to exotic (quantum field theoretic). In particular, much has been hypothesized about the possibility of some cognitive functions requiring the operation at a level of quantum physics. Experimental determination or even corroboration of such ideas as the Penrose-Hameroff Orchestrated Objective Reduction (Orch OR) theory [1] is currently being attempted using various experimental methods and instrumentation available to researchers.

Some fundamental questions in neuroscience can be addressed using the general principle of physics in conjunction with the known information about brain physiology at a level accessible to modern methods. For example, the metabolic rates of human brains have been studied extensively, and their energetic demands are well known. It is also known how much of the metabolic energy is required to carry out various molecular level processes such as protein production, motor protein motion, transcription and translation, etc. Finally, most of the brain's functional roles involve motor control, cognition, information storage, and processing. Physics has until recently treated information with some neglect focusing instead on entropy and its physically measurable manifestation, heat. Over 70 years ago, one of the most influential physicists of all time, Erwin Schroedinger, wrote a book entitled "What is Life" [2]. He outlined challenging problems plaguing the interpretation of biological processes using physical reasoning. Entropy reduction was a central fo- cal point of his discussion. While it is now known that entropy reduction that biological cells generate is at the cost of metabolic energy consumption and heat dissipation into the environment, a related problem of information storage and processing and the cost of such processes is still worth exploring.

We have now arrived at a time point in the history of science when exploring quantum phenomena and integrating quantum information science within the many subdisciplines of neurobiology is finally possible. Next-generation technologies for sensing, computing, modeling, and communicating are becoming available as advances in hardware, hybrid architectures, and quantum algorithms proceed rapidly. Indeed, quantum biology has shown great explanatory promise in many systems beyond photosynthetic lightharvesting complexes and avian magnetoreceptors, particularly in neurobiology (see Fig. 1, Ref. [3]). From this vantage point, quantum neurobiology should be based on concepts ranging from the most fundamental theory (quantum electrodynamics, quantum mechanics, and quantum chemistry of chromophores and fluorophores) to atomic rearrangements (cytochrome c oxidase and neuroreceptor/ion channel signaling) and biomolecular functionality (interaction of reactive oxygen species with mitochondrial membranes and cytoskeletal elements) to detailed neuronal studies in animals, connecting these insights up the organ hierarchy to Electroencephalogram (EEG), functional Magnetic Resonance Imaging (fMRI), and other brain imaging tools for quantum biomedicine applications. Breakthroughs await us in the area of quantum biology and especially in the ability of quantum concepts to provide explanations of numerous enigmatic features of biological systems such as synchronization, coherence, extraordinary sensory perception, e.g., in the case of olfaction or vision. Electromagnetic effects in neurobiology, which are particularly significant for magnetosensing may be implicated in the mitochondrial theory of 


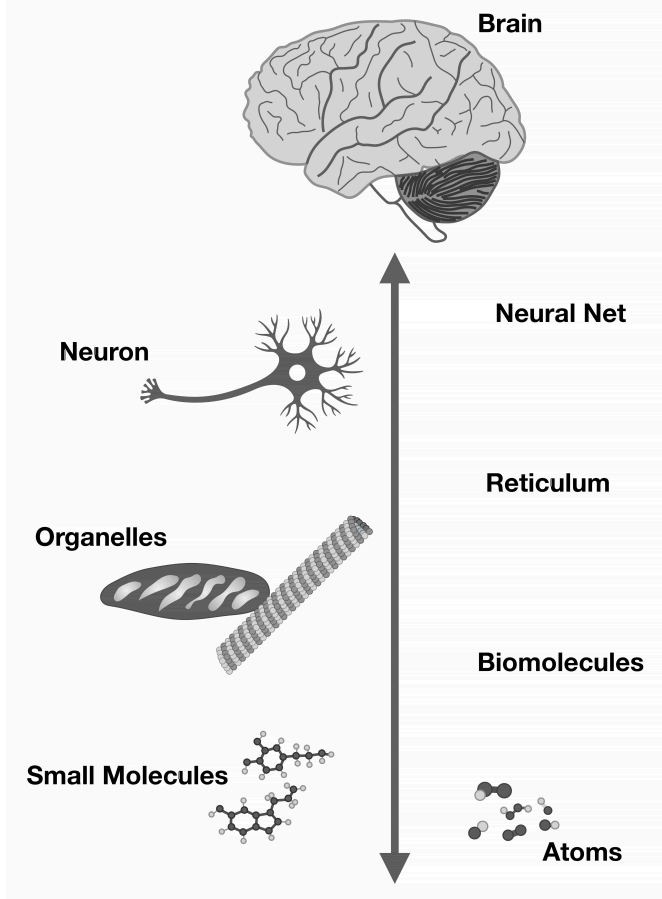

Quantum Fluctuations

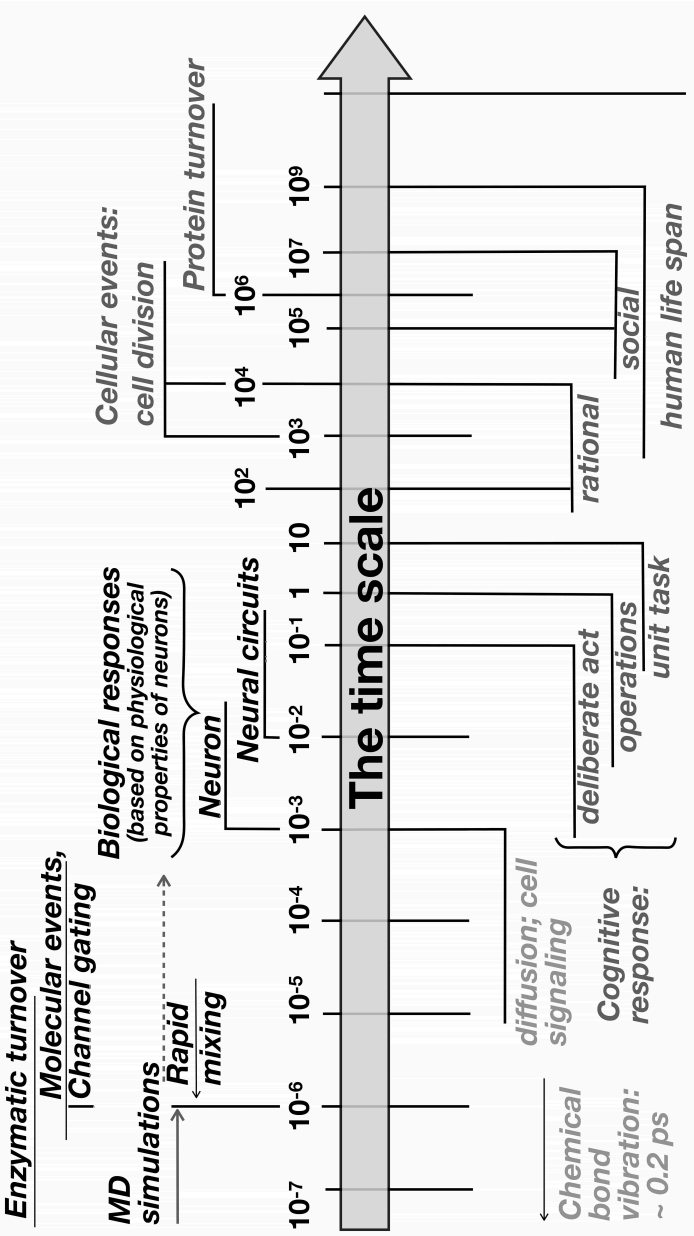

Fig. 1. Spatiotemporal hierarchy of the brain's complex dynamical organization spans multiple scales. The brain's cognitive hierarchy includes five or more layers across at least 12 orders of magnitude, from large (nearly brain-wide, $\sim 10 \mathrm{~cm}$ ), relatively slow $\left(<10^{2} \mathrm{~Hz}\right)$ processes based on neuronal membranes and synaptic network processes, downward and inward to increasingly smaller and faster molecular scales inside neurons, including the kilohertz $\left(10^{3}\right)$ to petahertz $\left(10^{15}\right)$ dynamics of cytoskeletal filaments, mitochondrial reticula, and their constituents at nanometer and sub-nanometer length scales. Adapted from [3].

aging, in human vision, in our circadian rhythms, and in the stimulation of the brain to improve symptoms of depression.

Beginning in 2006, quantum coherent states were observed in plant photosynthesis proteins in sunlight. Photons collected from the sun were shown to be converted to electronic excitations ('excitons') and propagating in superposition through 7 chlorophyll chromophores simultaneously to get to the reaction center to generate high-energy electrons for catalyzing biochemical synthesis [4]. The chromophores were largely non-polar 'pi resonance' ring structures, 'quantum-friendly' areas similar to phenyl, benzene and indole rings of aromatic amino acids in proteins and nucleic acids and lipid membranes. Quantum coherence among non-polar regions in geometrically constrained proteins, coherently pumped by ambient heat, had been predicted by Herbert Frohlich [5], suggesting quantum coherence might be widespread and entangled in non-polar regions within biomolecules (the 'quantum underground'). In such non-polar regions, anesthetic gases act by weak, non-specific quantum interactions and selectively block consciousness, sparing non-conscious brain activities [6]. Travis Craddock and Jack Tuszynski Stuart Hameroff [7] computationally demonstrated that quantum coherent states similar to those observed in chlorophyll could be generated in tubulin dimers. This prediction has not yet been measured experimentally but is a critical aspect that an ongoing experimental validation effort intends to investigate.

The objective behind this collection of papers is to present a glimpse into an integrated view of the human brain in terms of both its hierarchical structural organization and functional complexity. It is vital to develop an overview of modern neuroscience in a multi-scale approach, starting with molecular aspects and ending with psychological, psychiatric and pharmacological aspects. The assumptions of cognitive neuroscience employ abstractions from the theoretical constructs of 19th-century physics. Specifically, neural network models of "emergent conscious experience" rely on analogies to relations among molecules in crystals, fluids, and gases 
and employ chemical hypotheses regarding the emergence of thoughts, moods, and perceptions from chemical modulation of synaptic interactions among neurons. However, 20th and 21st-century science offer probabilistic perspectives from which to view the mind-brain nexus, including quantum entanglement processes that lower the entropy of the system [8]. Quantum mechanics and quantum field theory have given physicists extra "degrees of freedom", radically multiplied beyond thermodynamics. A relatively new offshoot of quantum physics is quantum information theory, quantum cryptography and actual quantum computation. Quantum logic formally upgrades the classical concept of a "bit" into the notion of a "qubit". Quantum interference permits vast computational parallelism. Cognitive paradigms drawing upon wave-like quantum logic have recently spawned new psychological literature. In 2013 a novel argument was advanced [9] that quantum modeling captures many empirically known relations among human perceptions, including mutual interference, order-dependence, and non-localized links, more accurately and entirely than does classical neural network theory. Werner Loewenstein extended quantum biology to include cognitive neuroscience [10]. Hence, it is high time to reconsider new quantum neuroscientific approaches developed over the past three decades as alternatives to traditionally dominant non-quantum paradigms. This has been paved by physicists like Hiroomi Umezawa, Kunio Yasue, Giuseppe Vitiello, and Travis Craddock, mathematicians like Roger Penrose, and biomedical investigators like Stuart Hameroff, Massimo Cocchi, Roman Poznanski and Gustav Bernroider. These innovators have laid a foundation for a possible rapprochement between material substrates and human cognition.

In the past few years, quantum-psychological and quantum-neurodynamical ideas have also provided alternative hypotheses concerning the genesis and nature of the mental illness. Several efforts in this direction were undertaken by members of the QPP (Quantum Psychopathology) initiative, an effort led by Massimo Cocchi to develop viable quantum paradigms of psychopathology [11]. Nancy Woolf and her collaborators suggested possible links between psychopathology and anomalous quantum computation in cytoskeletal proteins [12]. Paavo Pylkkanen hypothesized a relationship between diffuse physical substrates of mental illness and quantum "pilot waves" gone awry [13]. Massimo Cocchi and his collaborators [11] identified via membrane biophysics possible quantitative correlations between phospholipid composition, serotonin and quantum properties of the cytoskeleton in depression and psychosis. Paola Zizzi and Massimo Pregnolato suggested wave-like quantum logic as a possible non-Boolean algebra underlying the primary process in thought disorders [14]. Eliano Pessa and collaborators proposed a mathematical structure for psychiatric disease nosology based on symmetry breaking [15]. These ideas may guide future explorations of quantum paradigms of psychopathology. First, shifts from coherent to incoherent quantum brain states may, when aberrant, flag neural correlates of psychotic perception. Second, persistently mismatched phase relations among "parallel channels" of quantum information processing may shed light on clinical thought disorders. Third, bulk properties of brain states emerging from scaled-up quantum-statistical aspects of neural matter may include subjective experience, including normal and abnormal variation of moods. This may inform us about the effects of psychotherapeutic interventions, including electromagnetic brain stimulation. An important emerging topic is that of the gut-brain axis via microbiota [16].

It is entirely possible that no simple answers exist in this highly complex issue and that several molecular players perform different functions. Information processing may occur in a hybrid scenario where some classical operations are performed by microtubules (e.g., memory encoding). In contrast, some others (e.g., higher cognitive functions such as abstract reasoning) may involve ion channels operating quantum mechanically. We also wish to draw the reader's attention to signal amplification which may be critical to the integration of information processing across scales. Pertinent examples of molecular recognition and signal amplification of sensory inputs have been discussed by W. Lowenstein [10].

\section{Abbreviations}

Orch OR, orchestrated objective reduction; EEG, electroencephalogram; fMRI, functional magnetic resonance imaging; QPP, quantum paradigms in psychopathology.

\section{Author contributions}

MC, MD and JAT developed the concepts, wrote and edited the paper.

\section{Ethics approval and consent to participate}

Not applicable.

\section{Acknowledgment}

We thank all the participants in the two QPP conferences for their interesting and stimulating presentations and discussions. We particularly appreciate the lectures given by the following speakers: Massimo Cocchi, Gustav Bernroider, Lucio Tonello, Fabio Gabrielli, Jack Tuszynski, Natale Giuseppe Frega, Giovanni Lercker, Massimo Pregnolato, Giuseppe Vitiello, Marco A. Deriu, Marco Pettini, Brian Fertig, Ted Dinan, Paavo Pylkkanen, Francesco Cappello, Travis Craddock, Ursula Werneke, Alessandro Vercelli, Stuart Hameroff, Mark Rasenick and Hans Summhammer. The Organizing Committee consisted of: Massimo Cocchi, Jack Tuszynski, Marco A. Deriu and Marco Cavaglia.

\section{Funding}

Partial support for the organization of these conferences was provided by Bromatech, Società Italiana di Biologia Sperimentale and the Politecnico di Torino. 


\section{Conflict of interest}

The authors declare no conflict of interest. MC, MD and JAT are serving as Guest Editors of this journal. We declare that they had no involvement in the peer review of this article and has no access to information regarding its peer review. Full responsibility for the editorial process for this article was delegated to RRP.

\section{References}

[1] Hameroff S, Penrose R. Consciousness in the universe: A review of the 'Orch OR' theory. Physics of Life Reviews. 2014; 11: 39-78.

[2] Schrodinger E. What is life? With mind and matter and autobiographical sketches. Cambridge University Press: Cambridge. 2012.

[3] Craddock TJA, Kurian P, Tuszynski JA, Hameroff SR. Quantum Processes in Neurophotonics and the Origin of the Brain's Spatiotemporal Hierarchy. Neurophotonics and Biomedical Spectroscopy. 2019; 13: 189-213.

[4] Panitchayangkoon G, Hayes D, Fransted KA, Caram JR, Harel E, Wen J, et al. Long-lived quantum coherence in photosynthetic complexes at physiological temperature. Proceedings of the $\mathrm{Na}$ tional Academy of Sciences. 2010; 107: 12766-12770.

[5] Fröhlich H. Long-range coherence and energy storage in biological systems. International Journal of Quantum Chemistry. 1968; 2: 641-649.

[6] Craddock TJA, Kurian P, Preto J, Sahu K, Hameroff SR, Klobukowski M, et al. Anesthetic Alterations of Collective Terahertz Oscillations in Tubulin Correlate with Clinical Potency: Implications for Anesthetic Action and Post-Operative Cognitive Dysfunction. Scientific Reports. 2017; 7: 9877.
[7] Craddock TJA, Friesen D, Mane J, Hameroff S, Tuszynski JA. The feasibility of coherent energy transfer in microtubules. Journal of the Royal Society Interface. 2014; 11: 20140677.

[8] Poznanski RR, Cacha LA, Latif AZA, Salleh SH, Ali J, Yupapin P, et al. Theorizing how the brain encodes consciousness based on negentropic entanglement. Journal of Integrative Neuroscience. 2019; 18: 1-10.

[9] Wang Z, Busemeyer JR, Atmanspacher H, Pothos EM. The potential of using quantum theory to build models of cognition. Topics in Cognitive Science. 2013; 5: 672-688.

[10] Loewenstein, Werner R. The touchstone of life: molecular information, cell communication, and the foundations of life. Oxford University Press: Oxford. 1999.

[11] Tonello L, Massimo C. The cell membrane: is it a bridge from psychiatry to quantum consciousness? NeuroQuantology. 2010; 8: 54-60.

[12] Woolf NJ, Avner P, Tuszynski JA. Nanoneuroscience: structural and functional roles of the neuronal cytoskeleton in health and disease. Springer Science \& Business Media: Heidelberg. 2009.

[13] Pylkkänen, Paavo TI. Mind, matter and the implicate order. Springer Science \& Business Media: Heidelberg. 2006.

[14] Zizzi P, Massimo P. Quantum logic of the unconscious and schizophrenia. NeuroQuantology. 2012; 10: 566-579.

[15] Cocchi M, Gabrielli F, Pessa E, Pregnolato M, Tonello L, Zizzi P. Major depression and bipolar disorder: the concept of symmetry breaking. NeuroQuantology. 2012; 10: 676-687.

[16] Anderson SC, Cryan JF, Dinan T. The psychobiotic revolution: mood, food, and the new science of the gut-brain connection. $\mathrm{Na}$ tional Geographic Books: Washington. 2017. 